\title{
ANISOTROPY OF ELECTRIC CONDUCTIVITY AND DISSIPATION OF MAGNETIC FIELDS
}

\author{
M. KOPECKÝ \\ Astronomical Institute of the Czechoslovak Academy of Sciences, Ondřejov, Czechoslovakia \\ and

\section{KOPECKÝ} \\ Institute of Plasma Physics, Czechoslovak Academy of Sciences, Prague 9, Czechoslovakia
}

\begin{abstract}
The Joule dissipation of magnetic fields in the highest photosphere and spot layers can be considerably accelerated by the anisotropy of electric conductivity.
\end{abstract}

Most papers on magnetic field dissipation in the solar photosphere have assumed isotropic electric conductivity. Neglecting motion of the plasma, the magnetic field variation has then been described by the equation

$$
\frac{\partial \mathbf{H}}{\partial t}=\frac{c^{2}}{4 \pi \sigma} \Delta \mathbf{H},
$$

where $\sigma$ is the isotropic plasma conductivity. It is known, however, that a magnetic field may change a plasma into an anisotropic medium, and the conductivity anisotropy in a sunspot can be rather great depending upon the spot model, the intensity of the magnetic field, and the optical depth as was shown by Kopecký and Kuklin $(1966,1969)$. In considerations of the magnetic field dissipation, it is therefore necessary to utilize the equation that takes into account the influence of a magnetic field on conductivity. This fact has been noted already by Altschuler (1967), who came to the conclusion that the influence of the conductivity anisotropy is negligible. However, this conclusion holds only for the deeper layers of the photosphere and spots.

In a weakly ionized plasma $\left(n_{e} / n_{n} \ll 1\right)$, which the photosphere and spot may be considered to be, a change of the magnetic field is described by the equation derived by Cowling (1957). For the case considered it may be approximately written in the form

$$
\frac{\partial \mathbf{H}}{\partial t}=\frac{c^{2}}{4 \pi} \operatorname{rot}\left\{\frac{1}{\sigma_{0}} \operatorname{rot} \mathbf{H}+\frac{1}{c^{2} \alpha_{i n}}[\mathbf{H} \times[\operatorname{rot} \mathbf{H} \times \mathbf{H}]]+\frac{1}{e c n_{e}}[\operatorname{rot} \mathbf{H} \times \mathbf{H}]\right\}
$$

where $\sigma_{0}$ is the parallel conductivity and $\alpha_{i n}$ is the coefficient of friction between ions and neutral atoms. At the same time we assume that $v=0$ and that the terms containing the pressure gradients may be neglected.

Using (2) we obtain for a change of energy of the magnetic field

$$
\frac{\partial}{\partial t} \int \frac{H^{2}}{8 \pi} \mathrm{d} V=\frac{-1}{4 \pi} \int \mathrm{d} V\left\{\frac{c^{2}}{4 \pi \sigma_{0}}|\operatorname{rot} \mathbf{H}|^{2}+\frac{1}{4 \pi \alpha_{i n}}|[\operatorname{rot} \mathbf{H} \times \mathbf{H}]|^{2}\right\} .
$$


In the case of a force-free magnetic field it is obvious that the conductivity anisotropy does not play a role because electric currents flow along the magnetic field and hence the field dissipates according to Equation (1). In the opposite case (rot $\mathbf{H} \times \mathbf{H} \neq 0$ ) the dissipation increases approximately $\alpha$ times, where

$$
\alpha \equiv \frac{\sigma_{0}}{\sigma_{\perp}}=1+\frac{H^{2} \sigma_{0}}{c^{2} \alpha_{i n}} .
$$

For the solar photosphere and spots the coefficient of anisotropy $\alpha$ takes values much greater than unity only in the highest layers as may be seen in Table I, where the values of $\alpha$ are determined for the chemical composition used by Zwaan (1965), and the effective cross sections are those considered in the paper by Kopecký and Kuklin (1969) at $r_{i n}^{2}=10^{-15} \mathrm{~cm}^{2}$.

\section{TABLE I}

Approximate values of the anisotropy coefficient $\alpha$ of electric conductivity

\begin{tabular}{llll}
\hline $\begin{array}{l}H= \\
\log P_{g}\end{array}$ & 300 & 1000 & 3000 \\
\hline 3.0 & 9.02 & $1.13 \times 10^{2}$ & $1.01 \times 10^{3}$ \\
4.5 & 1.01 & 1.11 & 2.03 \\
6.0 & 1.00 & 1.00 & 1.00 \\
\hline
\end{tabular}

In these highest layers of the spots and photosphere the anisotropy of electric conductivity can thus essentially lessen the time of the Joule dissipation of magnetic fields up to one or two orders. Therefore, in these highest photospheric layers, magnetic fields which are not force-free should dissipate more rapidly than they have been assumed to do until now.

In the first place this effect should appear in a relatively rapid dissipation of inhomogeneities of the magnetic field within sunspots. Thus, for example, for bright points in a large umbra with observed lifetimes of $10^{3}$ to $10^{4} \mathrm{~s}$, the time for Joule dissipation is $10^{3}$ to $10^{5} \mathrm{~s}$ at $\sigma_{0}=10^{9}$ to $10^{11} \mathrm{CGSE}$ (Kopecký and Obridko, 1968). If the electric conductivity is anisotropic with $\alpha=10$ to $10^{2}$, their Joule dissipation is thus completely comparable with the observed lifetime.

We can see to what extent $\sigma_{0}$ can differ from $\sigma_{\perp}$ in some spot models from Figure 1 , where the dependence of $\log \sigma_{0}$ (solid lines) and $\log \sigma_{\perp}$ at $H=3000 \mathrm{G}$ (dashed lines) on the optical depth $\tau$ is given for the case of the spot models by Van 't Veer (1963) with an umbral area $A=50,80$, and 110 millionths of the Sun's hemisphere (Kopecký and Kuklin, 1966). Figure 1 shows, first of all, a considerable dependence of the anisotropy of electric conductivity, and therefore the characteristic dissipation time, on the physical conditions in a spot as a consequence of a change of its area.

The Joule dissipation of magnetic fields in the highest photosphere and spot layers can thus be considerably accelerated by the anisotropy of electric conductivity. This again gives evidence for the fact that the dissipation processes cannot be completely neglected in hydromagnetic considerations of processes in the observed layers of the 


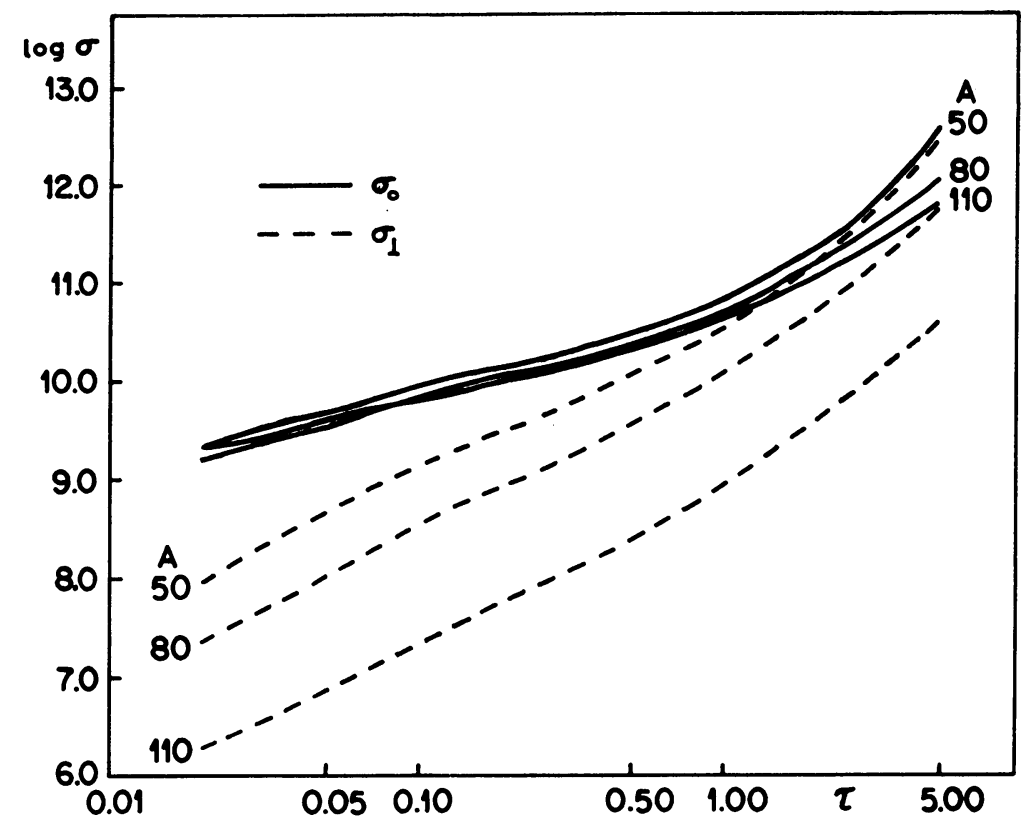

Fig. 1. The dependence of $\log \sigma_{0}$ and $\log \sigma_{\perp}$ depth $\tau$ for the case of the spot models by Van 't Veer with the umbra area $A=50,80$ and 110 millionths of the Sun hemisphere.

Sun. Above all the dissipation processes cannot be neglected in considerations of the generation of magnetic fields as, for example, in the theory by Getling and Tversky (1968), where invariably currents with a component perpendicular to the magnetic field are generated, so that the generated fields are not force-free, and therefore characteristic dimensions of the field generation and dissipation processes are comparable.

\section{References}

Altschuler, M. D.: 1967, Solar Phys. 1, 377.

Cowling, T. G.: 1957, Magnetohydrodynamics, Interscience Publishers, London.

Getling, A. V. and Tversky, B. A.: 1968, Astron.Zh. 45, 606.

Kopecký, M. and Kuklin, G. V.: 1966, Bull. Astron. Inst. Czech. 17, 45.

Kopecký, M. and Kuklin, G. V.: 1969, Solar Phys. 6, 241.

Kopecký, M. and Obridko, V.: 1968, Solar Phys. 5, 354.

Zwaan, C.: 1965, Rech. Astron. Observ. Utrecht 12, (4). 